\title{
Effect of starch-meal chilling and reheating on postprandial glycaemia in overweight and obese individuals
}

\author{
A. Alzaabi, B.A. Fielding and M.D. Robertson \\ Department of Nutritional Sciences, Faculty of Health and Medical Sciences, University of Surrey, Guildford, GU2 \\ $7 W G$
}

Both the amount and type of dietary carbohydrates contribute to glycaemia and insulinaemia. Normally, in healthy people, the secretion of insulin maintains plasma glucose levels within a narrow range. However, in insulin resistance, inefficient insulin action impairs fasting and postprandial glucose regulation ${ }^{(1)}$. Insulin resistance has been associated with the development of chronic metabolic diseases such as type 2 diabetes mellitus and $\mathrm{CVD}^{(2)}$. Several studies have demonstrated that replacing digestible carbohydrates with indigestible ones reduces postprandial glucose responses. A pilot study in our laboratory indicated that chilling and reheating a pasta meal significantly lowered the glycaemic response in healthy, normal weight adults compared to identical meals served freshly cooked, potentially due to the formation of type 5 resistant $\operatorname{starch}^{(3)}$. Accordingly, the objective of this study was to determine whether domestic chilling and reheating would have a similar effect, of reducing postprandial glycaemia in overweight and obese individuals.

Ten overweight and obese participants (6 females) with mean BMI of 29.6 (SD 4.2) were recruited for the study. The study was conducted as a randomised cross-over single-meal study. The trial involved two different test meals; a freshly cooked meal (control) and a chilled and reheated meal (intervention) in the form of pasta, olive oil and tomato passata. These two meals were each consumed on a different occasion with a wash-out period of one week. Each meal consisted of $11 \mathrm{~g}$ fat, $75 \mathrm{~g}$ carbohydrate and $13.5 \mathrm{~g}$ protein, providing $1950 \mathrm{KJ}$. Postprandial capillary blood sampling for glucose and insulin was undertaken at baseline and then at $15,30,45,60,90$ and 120 minutes after meal consumption.

In a post-hoc analysis, participants were considered in two groups; insulin sensitive $(n=5)$ and insulin resistant $(n=5)$ stratified by fasting insulin sensitivity (HOMA2 > or <1.0). In the insulin sensitive group, the mean glucose iAUCs were 134 (SD 90 ) v 167 (SD 91 ) mmol. $1^{-1} .120$ min for control $\mathrm{v}$ intervention meals respectively $(t(4)=-8 \cdot 902, p=0 \cdot 001)$. However, in contrast, there was no statistically significant difference found for the postprandial glucose response in the insulin resistant group (mean glucose iAUCs were 210.30 (SD 44.39) v 193.04 (SD 74.92) mmol. $1^{-1} .120$ min for control v intervention). There was no significant between group effect

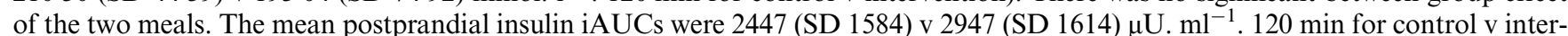
vention in the insulin sensitive group (ns), and 6106 (SD 2006) v 5269 (SD 1905) $\mu \mathrm{U} \mathrm{ml}^{-1} .120$ min for control v intervention in the insulin resistant group (ns). A statistically significant difference between group effect of the two meals for plasma insulin was noted ( $t$ $(8)=2 \cdot 529, p=0 \cdot 035)$.

In conclusion, the analysis revealed a difference between the groups in the postprandial insulin response after ingestion of the treatment meals but no effect on plasma glucose. This positive response in insulin might be favourable in the management of hyperinsulinaemia for those who are insulin resistant. This study is continuing further recruitment to obtain more statistical robust results.

1. Robertson MD. (2012) Dietary-resistant starch and glucose metabolism. Curr Opin Clin Nutr 15(4), 362-7.

2. Johnston KL, Thomas EL, Bell JD, Frost GS, Robertson MD. (2010) Resistant starch improves insulin sensitivity in metabolic syndrome. Diabetic Med 27(4), 391-7.

3. BBC Website. Trust Me, I'm a Doctor - Can my leftovers be healthier than the original meal? [cited]. Available from: http://www.bbc.co.uk/programmes/articles/3LncBcDcCXKgtpFvrDZVnNQ/can-my-leftovers-be-healthier-than-the-original-meal. 